\title{
CXCR7 correlates with the differentiation of hepatocellular carcinoma and suppresses HNF4 $\alpha$ expression through the ERK pathway
}

\author{
TONG-CHUN XUE ${ }^{1,2}$, QING-AN JIA ${ }^{1,2}$, YANG BU $^{1,2}$, RONG-XIN CHEN $^{1,2}$, \\ JIE-FENG CUI ${ }^{1,2}$, ZHAO-YOU TANG ${ }^{1,2}$ and SHENG-LONG YE ${ }^{1,2}$ \\ ${ }^{1}$ Liver Cancer Institute, Zhongshan Hospital, Fudan University, Shanghai; ${ }^{2}$ Key Laboratory of Carcinogenesis \\ and Cancer Invasion, Fudan University, Ministry of Education, Shanghai, P.R. China
}

Received April 16, 2014; Accepted June 12, 2014

DOI: 10.3892/or.2014.3501

\begin{abstract}
Hepatocellular carcinoma (HCC) is a malignancy with dysregulated differentiation. However, effective differentiation therapy for HCC is lacking. Previous evidence suggests that CXCR7 is associated with the differentiation of embryonic stem cells. Here, we evaluated the potential role of CXCR7 in the differentiation of HCC. In HCC cell lines, the expression of cancer stem cell-related markers was assessed by flow cytometry and confirmed by western blot and immunofluorescence analyses. Dimethyl sulfoxide, oncostatin M and dexamethasone were used to induce the differentiation of HCC. Immunohistochemical assay was performed on a tissue microarray based on $112 \mathrm{HCC}$ cases that received hepatectomy. Ligand activation, inhibition assays and RNA interference were used to analyze the regulation of hepatocyte nuclear factor $4 \alpha$ (HNF4 $\alpha$ ) by the CXCR7 pathway. Huh7 and HCCLM3 cell lines were screened for differentiation induction based on biomarkers of hepatic cancer stem cells. CXCR7 was found to be closely associated with the differentiation of HCC, and an inverse expression trend between CXCR7 and HNF4 $\alpha$ was found upon induced differentiation. Clinically, high CXCR7 expression was negatively correlated with $\mathrm{HNF} 4 \alpha$ expression in patients with relatively well-differentiated HCC. Moreover, high CXCR7 expression was correlated with poor overall survival and accelerated post-resection metastasis in HCC with a low HNF4 $\alpha$ level. Mechanistically, CXCR7 signaling inhibited HNF4 $\alpha$ through extracellular regulated protein kinase (ERK) activation, which was inhibited by U0126, an inhibitor of MAPK/ERK kinases 1 and 2. Knockdown of CXCR7 further confirmed that CXCR7 signaling can regulate
\end{abstract}

Correspondence to: Professor Sheng-Long Ye, Liver Cancer Institute, Zhongshan Hospital, Fudan University, 180 Fenglin Road, Shanghai 200032, P.R. China

E-mail: slye@shmu.edu.cn

Key words: CXCR7, differentiation, hepatocellular carcinoma, ERK, hepatocyte nuclear factor $4 \alpha$
HNF4 $\alpha$ expression. Taken together, our findings indicate that CXCR7 participates in the differentiation of HCC by regulating HNF4 $\alpha$. The CXCR7-ERK-HNF4 $\alpha$ cascade represents a new target for the differentiation therapy of HCC.

\section{Introduction}

Hepatocellular carcinoma (HCC) is a malignancy with dysregulated differentiation (1). Differentiation-related genes in HCC have been enumerated; for example, the well-known biomarker $\alpha$-fetoprotein (AFP), only expressed during early stages of liver development, is always highly expressed in HCC. Differentiation therapy has been quite successful for various malignancies, particularly acute promyelocytic leukemia (2). However, effective differentiation therapy is lacking for $\operatorname{HCC}(3,4)$, partly due to the limited knowledge of dysregulated differentiation in HCC (5).

During normal liver development, key transcription factors, including hepatocyte nuclear factor $4 \alpha(\mathrm{HNF} 4 \alpha), \mathrm{HNF} 1 \alpha$, HNF1 $\beta$, CCAAT/enhancer binding protein $\alpha(\mathrm{C} / \mathrm{EBP} \alpha)$ and $\mathrm{C} /$ $\operatorname{EBP} \beta$, control differentiation (6). HNF4 $\alpha$ plays a critical role in hepatocyte differentiation (7) and controls the expression of more than $40 \%$ of hepatocyte genes. Recently, the critical roles of HNF4 $\alpha$ in the dysregulated differentiation and carcinogenesis in HCC have been identified $(8,9)$. A nude mouse model of $\mathrm{HCC}$ showed that a recombinant adenovirus carrying $\mathrm{HNF} 4 \alpha$ potently promoted the differentiation of $\mathrm{HCC}$ into normal hepatocytes and suppressed tumorigenesis (4).

CXCR7 was formerly known as RDC1 or orphan receptor since its ligands were unknown. Knowledge concerning CXCR7 increased after the functional ligands SDF-1 $\alpha$ (or CXCL12) and ITAC (or CXCL11) were found (10,11). CXCR7 is a G-protein-coupled seven-transmembrane receptor. However, it also mediates $\beta$-arrestin-biased signaling (12). Functionally, CXCR7 has been shown to be involved in cardiac development (13). In addition, evidence suggests links between CXCR7 and tumor proliferation or invasion (14). Moreover, our previous research demonstrated the critical role of CXCR7 in HCC $(15,16)$, which is related to differentiation (unpublished data). Additionally, during the differentiation of embryonic stem cells, the epigenetic suppression of CXCR7 by SUZ12 
is lost, and the expression of CXCR7 can be increased 20 -fold (17), suggesting a critical role for CXCR7 in stem cell differentiation. Therefore, we directed our attention to the potential role of CXCR7 in the dysregulation of the differentiation in $\mathrm{HCC}$.

Here, CXCR7 was identified to be closely associated with the differentiation of HCC, and a close relationship between CXCR7 and HNF4 $\alpha$ was found, which was confirmed by the immunohistochemical (IHC) staining of tissue microarrays (TMAs). High CXCR7 levels and low HNF4 $\alpha$ levels were correlated with poor survival. Furthermore, ligand activation, inhibition assays, and RNA interference (RNAi) demonstrated that the regulation of HNF4 $\alpha$ by CXCR7 was mitogen-activated protein kinase (MAPK)-dependent.

\section{Materials and methods}

Reagents and antibodies. Oncostatin M (OSM), SDF-1 $\alpha$ and CXCL11 were purchased from Peprotech (Rocky Hill, NJ, USA). U0126 and dimethyl sulfoxide (DMSO) were purchased from Sigma-Aldrich (St. Louis, MO, USA).

Monoclonal antibodies used in the flow cytometric analysis were mouse anti-human monoclonal antibodies (mAbs) CD44-PE, CD133-APC, CD90-PE, CD24-FITC, the IgG-PE isotype, the IgG-APC isotype and the IgG-FITC isotype (all purchased from Miltenyi Biotec, Bergisch Gladbach, Germany). Antibodies used for immunofluorescence, immunoblotting and immunohistochemistry were as follows: mouse anti-human monoclonal CD90 (Abcam, Cambridge, MA, USA), rabbit anti-human polyclonal CD133 (Abnova, Walnut, CA, USA), mouse anti-human monoclonal CD44, rabbit anti-human albumin (ALB), phospho-p44/42 MAPK (Erk1/2), and rabbit anti-human HNF4 $\alpha$ mAb (Cell Signaling Technologies, Danvers, MA, USA), rabbit anti-human polyclonal CD24, rabbit anti-human $\beta$-actin mAb (Epitomics, Burlingame, CA, USA), rabbit anti-human polyclonal transferrin (TF) (Proteintech Group Inc., Chicago, IL, USA), rabbit anti-human AFP mAb, rabbit anti-human C/EBP $\alpha$ and $\mathrm{C} /$ EBP $\beta$ mAb, Erk2/p42 MAPK antibody (Epitomics), rabbit anti-human CXCR7 IgG (Novus Biologicals, Littleton, CO, USA), and horseradish peroxidase-conjugated goat anti-rabbit IgG F(ab')2 antibodies (Jackson ImmunoResearch, West Grove, PA, USA).

Cell lines and culture. Human HCC cell lines with elevated lung metastatic potential (MHCC97L, MHCC $97 \mathrm{H}$, and HCCLM3) were established at the Liver Cancer Institute of Fudan University. The human HCC cell lines with low metastatic potential were SMMC-7721 (established at the Second Military Medical University), Huh7 and HepG2 (obtained from the American Type Culture Collection). These cell lines were cultured in high-glucose DMEM (Gibco-BRL, Grand Island, NY, USA) supplemented with $10 \%$ fetal bovine serum (Hyclone, Logan, UT, USA).

To induce differentiation, 10 to $40 \mathrm{ng} / \mathrm{ml}$ OSM and 1 or $5 \mu \mathrm{M}$ dexamethasone (Dex) were used. Chemical differentiation inducer DMSO was also used to help establish a model of differentiation induction. For the CXCR7 stimulation assay, $100 \mathrm{ng} / \mathrm{ml}$ recombinant human SDF- $1 \alpha$ and $200 \mathrm{ng} / \mathrm{ml} \mathrm{CXCL11}$ were used. For the inhibition assay, cells were serum-starved for at least $8 \mathrm{~h}$ before the MEK1/2 inhibitor U0126 (10 $\mu \mathrm{M})$ was added.

Flow cytometry. The expression levels of cancer stem cell (CSC)-related markers were determined by flow cytometry. Briefly, tumor cells were grown to $80 \%$ confluency. After trypsin digestion, the cells were re-suspended in medium at a concentration of $1 \times 10^{6}$ cells $/ \mathrm{ml}$ and incubated with antibodies against CD44, CD133, CD90 and CD24 (diluted 1:11) at $4^{\circ} \mathrm{C}$ for $15 \mathrm{~min}$. After washing 3 times with PBS, the cells were analyzed using a FACS flow cytometer (BD Biosciences, Franklin Lakes, NJ, USA).

Immunofluorescence. Cell surface expression of CSC-related markers and CXCR7 was also determined by immunofluorescence analysis. Cells were grown on glass coverslips to $40-50 \%$ confluency and fixed, permeabilized, blocked, and incubated with primary monoclonal antibodies overnight at $4^{\circ} \mathrm{C}$. Slides were washed and incubated with an anti-mouse or an anti-rabbit Cy3-conjugated secondary antibody (Jackson ImmunoResearch). Cells were counterstained with 4-6-diamidino-2-phenylindole to visualize cell nuclei and for inspection by fluorescence microscopy (Olympus).

Western blot analysis. Western blotting was performed according to the wet transfer protocol using the Bio-Rad Transfer Cell System (Bio-Rad, Ontario, Canada). Analyses of protein expression were performed according to the manufacturer's instructions. Images were examined using Image Lab Software ${ }^{\circledR}$ (Bio-Rad).

RNA interference. Small interfering RNAs (siRNAs; GenePharma Corp., Shanghai, China) demonstrated to be effective for the knockdown of CXCR7 expression (15) were used. siRNA transfection of the cells was performed using the Lipofectamine 2000 protocol (18). A negative control siRNA, 5'-UUC UCC GAA CGU GUC ACG UTT-3', was also used. A FAM-labeled negative control siRNA was used to monitor transfection efficiency.

Gene microarray. The Oligo GEArray Human Chemokine and Chemokine Receptors Chip (SuperArray Bioscience, Frederick, MD, USA) was used to compare profiles of the HCCLM3, MHCC97-L, and SMMC-7721 cells according to the manufacturer's protocol. Total-RNA was extracted, isolated, and purified according to the TRIzol reagent (Invitrogen Corp., Carlsbad, CA, USA) protocol. GEArray ${ }^{\circledR}$ Analyzer Software (SuperArray Bioscience) was used for data analysis. The microarray analysis was performed twice with similar results.

Patients and follow-up. Ethical approval was obtained from the Zhongshan Hospital Research Ethics Committee, and informed consent was obtained from each patient. Data from 112 patients were retrieved from the prospectively designed database. These patients underwent hepatectomy by the same surgical team from January 2000 to May 2004. The hepatectomy for HCC was carried out as described previously (19). All patients were classified as Child-Pugh A, and all tumors were identified as HCC by histological analysis. 
A

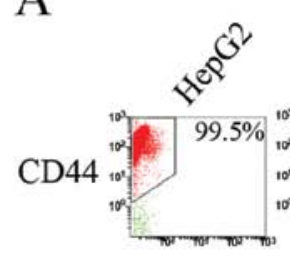

CD133 $13^{102}$

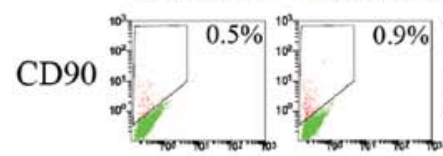

$\mathrm{CD} 24$

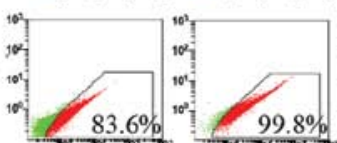

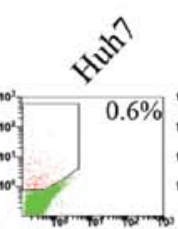

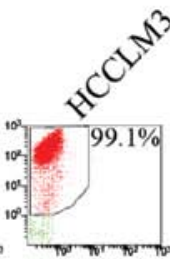

$72.6 \%$
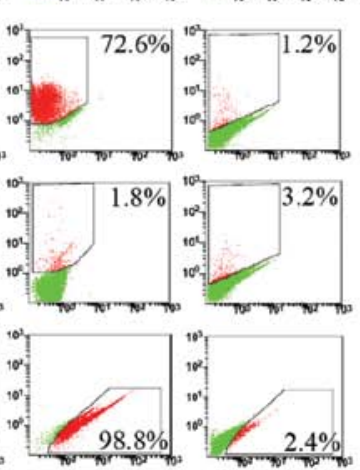

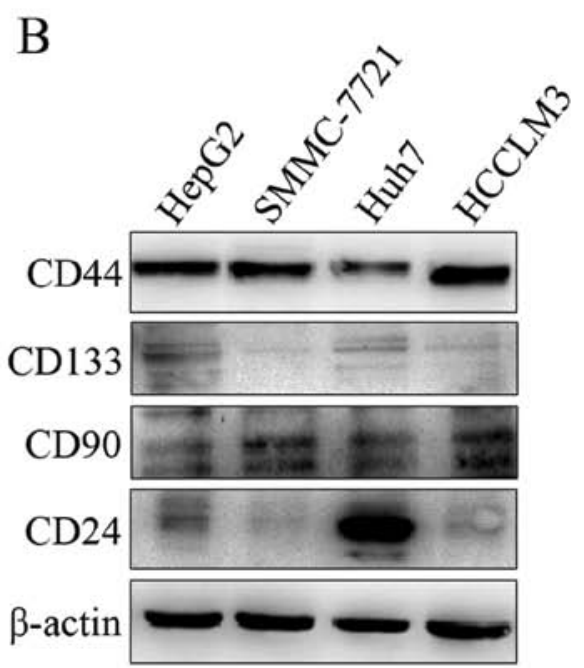

$\mathrm{C}$
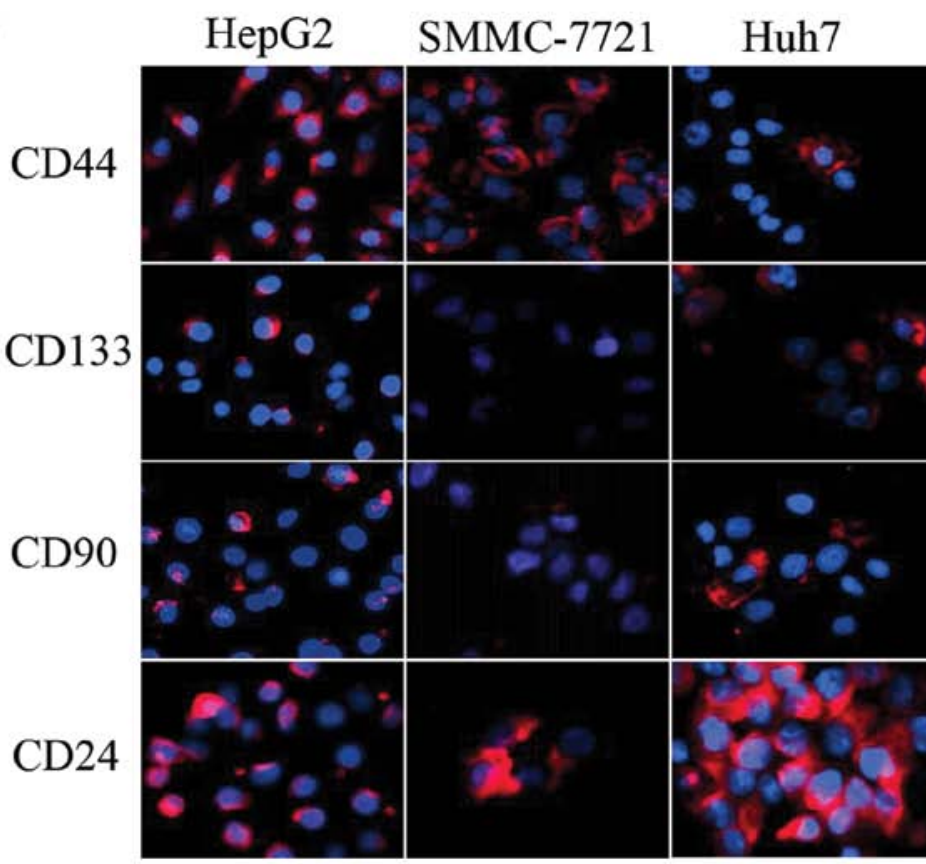

HCCLM3

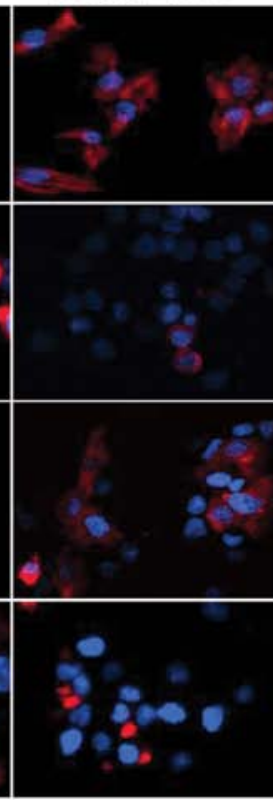

Figure 1. CSC-related biomarkers in the HCC cell lines. (A) Flow cytometry indicated that the Huh7 cells had a relatively high ratio of CD133- and CD24positive cells, whereas the HCCLM3 cells had a relatively high ratio of CD90- and CD44-positive cells. (B) Western blotting showed that the expression levels of these biomarkers were similar to the flow cytometric findings; particularly strong CD24 expression was noted in the Huh7 cells. (C) Immunofluorescence further confirmed the results of the flow cytometry; particularly CD90 expression in the HCCLM3 cells and CD24 expression in Huh7 cells.

Regular follow-up procedures in our clinic include the following: AFP assay and liver ultrasonography every 3 months during the first year and every 6 months thereafter; and magnetic resonance imaging or computed tomography scanning after 1 month and every 6 months thereafter. Chest computed tomography scanning is regularly used to identify lung metastasis. Lung metastasis was confirmed by biopsy through endoscopy or histology after partial pulmonary resection. Until May 2009, lung metastasis was found in 56 patients. Ten patients with resectable lung metastases received partial resection of the lung.

TMA and IHC. Hematoxylin and eosin-stained slides were screened for optimal tumor content and tissue adjacent to the tumor (TAT) with a distance of $2 \mathrm{~cm}$. The TMA was constructed in accordance with standard procedures (20) based on 112 tumor tissues and 46 TATs. Two cores were taken from each formalin-fixed, paraffin-embedded HCC sample by using punch cores that measured $1.0 \mathrm{~mm}$ in diameter from the center of the tumor foci and TAT. A two-step method of IHC including heat-induced antigen-retrieval procedure was performed as previously described (20). Detection without the primary antibody was considered as the negative control.

Scoring and categorization of CXCR7 expression. CXCR7 expression was defined by staining intensity and the percentage of positive tumor cells as described previously (21). Two pathologists observed the results independently. We simplified 
A

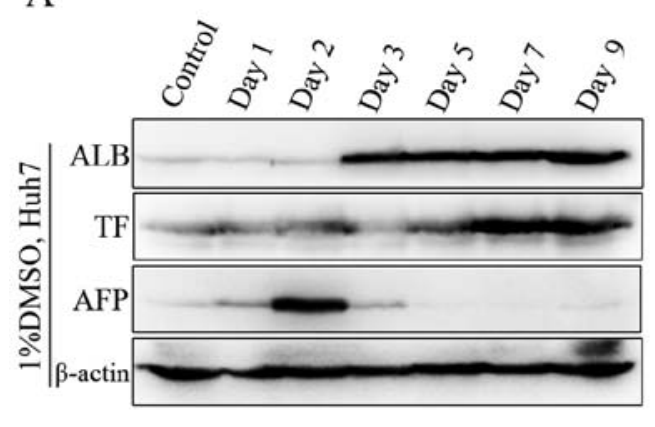

B

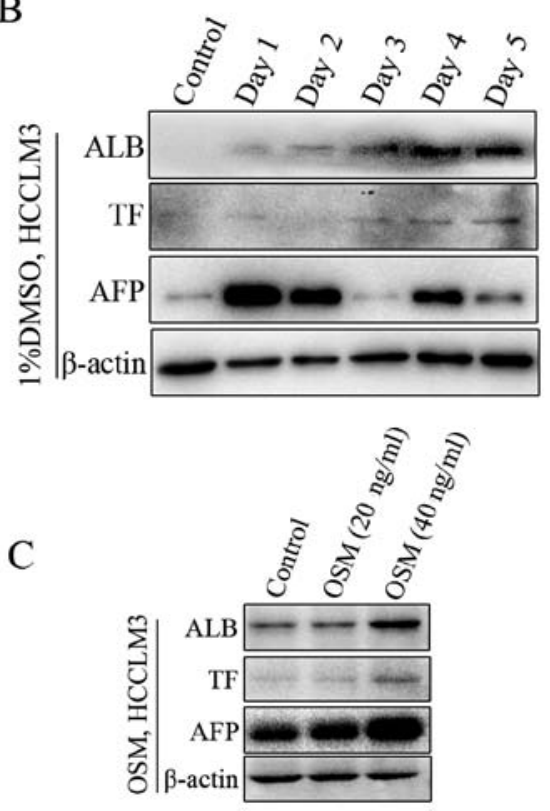

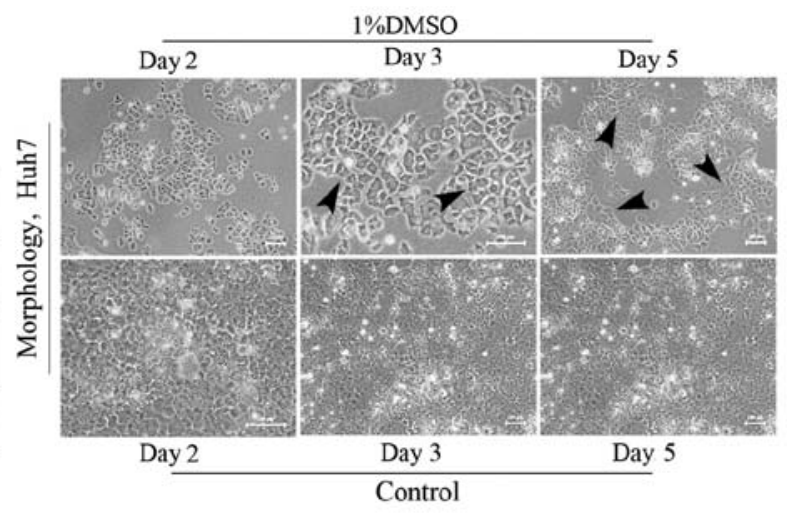
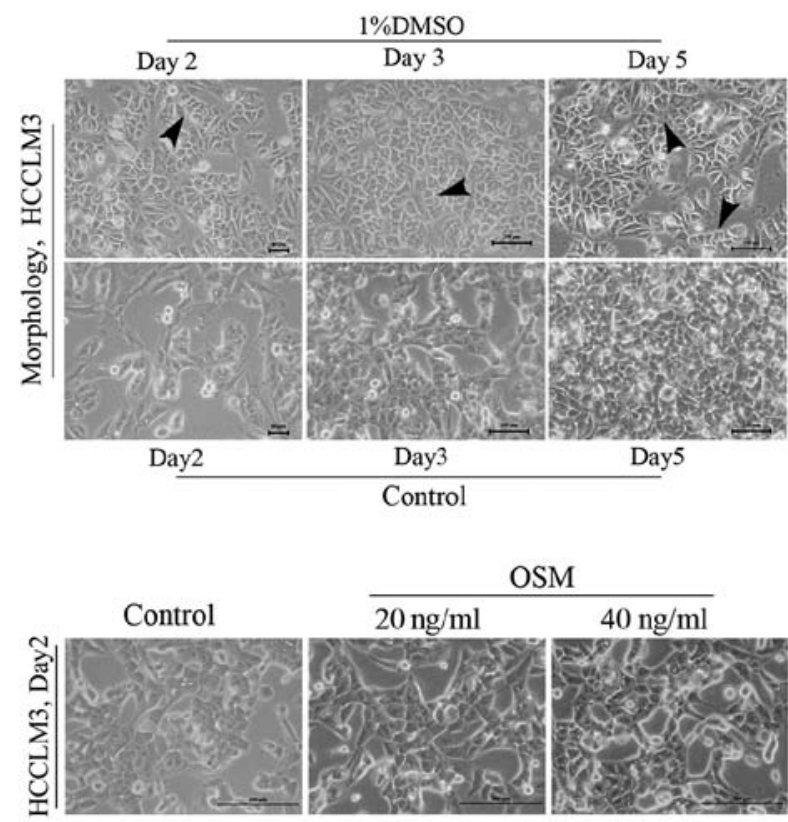

Figure 2. Differentiation of HCC cells to hepatocyte-like cells in vitro. (A) The Huh7 cells were induced to differentiate by $1 \%$ DMSO treatment. Western blot analysis indicated increased albumin (ALB) and transferrin (TF). AFP increased at day 2 and gradually decreased. Microscopically, the typical morphological features of differentiation were noted from the early stages of differentiation, before the upregulation of plasma proteins. Black arrows indicate a characteristic cubical shape and cell borders were enhanced in response to DMSO treatment. (B) HCCLM3 cells were induced to differentiate by $1 \%$ DMSO treatment. Similar to the Huh7 cells, HCCLM3 cells showed increased ALB and TF and the morphological features of hepatocyte-like cells are indicated by black arrows. (C) Cells were treated with OSM. Elevated expression levels of both ALB and TF were observed in the HCCLM3 cells treated with OSM in a dose-dependent manner, although no extraordinary morphological features were observed. Images were captured (under x 200 magnification) using phase-contrast imaging.

the assay results of CXCR7 into $\mathrm{CXCR}^{\text {Low }}$ (weak staining) and CXCR7 $7^{\text {High }}$ (strong staining). Similarly, HNF4 $\alpha$ expression was categorized as $\mathrm{HNF} 4 \alpha^{\text {Low }}$ or HNF4 $\alpha^{\text {High }}$, except that $\mathrm{HNF} 4 \alpha^{\mathrm{Low}}$ included the negatively stained population due to the generally weak staining.

Statistical analysis. When two groups of cells or tissues were compared, analysis was performed with the Student's t-test. The Pearson $\chi^{2}$ test was used to compare qualitative variables in the clinical pathology analysis. When expected sample values were $<5$, Fisher's exact test was used. Spearman's rank test was used to detect the correlation between variables. Overall survival (OS), time to progression (TTP), and time to extrahepatic metastasis were observed in the survival analysis. OS was calculated from the date of hepatectomy to the date of death regardless of cause. TTP was calculated from the date of hepatic resection to the date of recurrence. Time to lung metastasis was calculated from the date of hepatectomy to the date of lung metastasis with definite clinical diagnosis. The patients lost in the follow-up and the patients who had not achieved the desired results at the end of this study were recognized as censored cases. The Kaplan-Meier method was used to describe the survival curves, and the log-rank test was used to compare survival distributions between groups. The Breslow test was also used when survival curves indicated greater differences during the early follow-up period. All P-values were obtained using two-tailed tests and the statistical significance was set at 0.05 . Statistical analyses were carried out by SPSS 18.0 Software (SPSS Inc., Chicago, IL, USA).

\section{Results}

Screening of the HCC cell lines for differentiation induction. Due to the highly heterogenetic character of HCC, we first detected HCC-related stem cell biomarkers in four HCC cell lines (HepG2, SMMC-7721, Huh7 and HCCLM3) with 


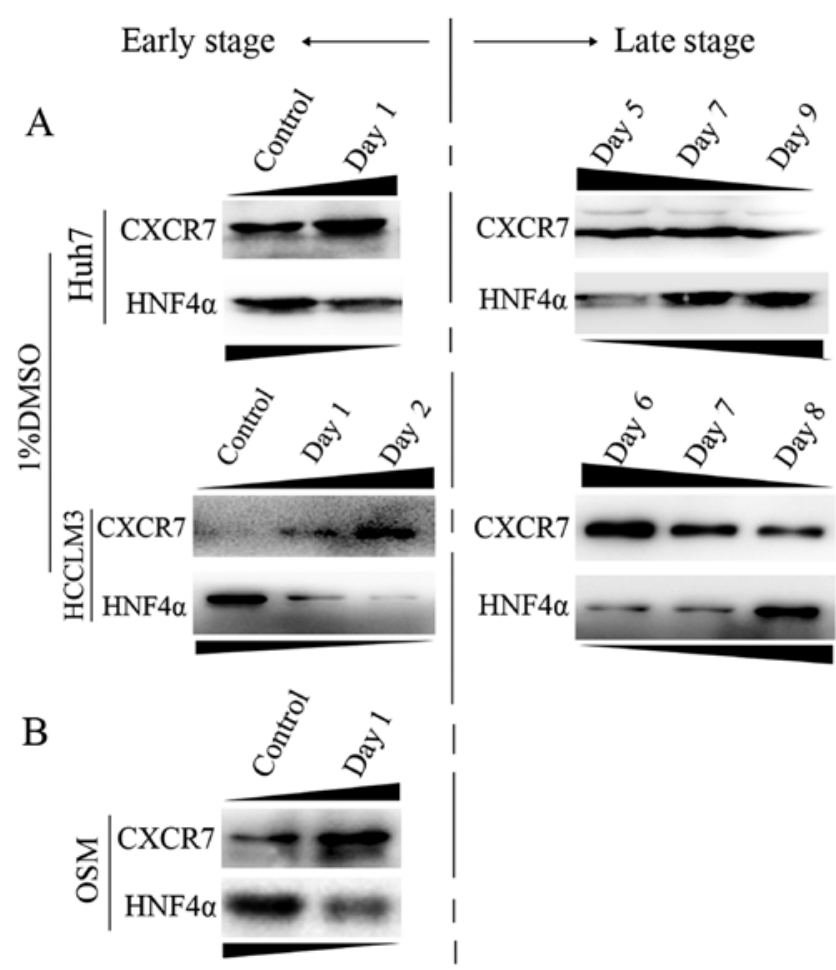

Figure 3. CXCR7 is inversely correlated to HNF4 $\alpha$ upon induced differentiation. (A) Huh7 and HCCLM3 cells were treated with $1 \%$ DMSO, respectively. The expression level of CXCR7 was elevated, and the HNF4 $\alpha$ level was decreased during the early stage of induced differentiation as observed by western blotting. During the late stage of induced differentiation, the level of CXCR7 was decreased and the HNF4 $\alpha$ level was elevated. (B) HCCLM3 cells were treated with OSM. The CXCR7 level was elevated while the opposite trend was observed with $\mathrm{HNF} 4 \alpha$ during the early stage of differentiation in response to $40 \mathrm{ng} / \mathrm{ml}$ OSM treatment.

different characteristics and backgrounds. Flow cytometric analysis indicated that Huh7 cells had the highest ratios of CD133 (72.6\%) and CD24 (98.8\%), which was confirmed by western blot and immunofluorescence analyses (Fig. 1). HCCLM3 cells had the highest CD90-positive ratio (3.2\%) and a CD44-positive ratio of $>90 \%$. Since these CSC-related markers have been linked to HCC progression and dysregulated differentiation potential (22-24), Huh7 and HCCLM3 cells were selected for further analysis.

HCC cells differentiate into hepatocyte-like cells upon induced differentiation. The differentiation induction model for HCC was established using potential differentiation inducers. DMSO was used as a chemical inducer of differentiation. OSM, which has the ability to maintain the maturation and differentiation capacity of hepatocytes (25), was also used. Both in the Huh7 and in the HCCLM3 cells, DMSO showed a strong ability to induce HCC differentiation into normal hepatocytes, based on hepatocyte differentiation markers or elevated expression of plasma proteins ALB and TF, and the typical morphological features of differentiated hepatocytes (Fig. 2A and B). The typical morphology of differentiation was maintained from day 2 until late stages of the induction process. Meanwhile, results from HCCLM3 cells revealed that OSM had the ability to induce elevated expression of ALB and TF (Fig. 2C). However, no typical morphological features were noted, which indicated the main role of OSM in maintaining the maturation
Table I. Clinicopathological factors and combined CXCR7 and HNF4 $\alpha$ expression.

\begin{tabular}{|c|c|c|c|}
\hline \multirow[b]{2}{*}{$\begin{array}{l}\text { Clinicopathological } \\
\text { characteristics }\end{array}$} & \multicolumn{2}{|c|}{ No. of patients } & \multirow[b]{2}{*}{ P-value } \\
\hline & $\begin{array}{c}\text { CXCR7 }^{\text {High/ }} \\
\text { HNF4 } \alpha^{\text {Low }} \\
(\mathrm{n}=30)\end{array}$ & $\begin{array}{c}\text { CXCR7 }^{\text {Low }} / \\
\text { HNF } 4 \alpha^{\text {High }} \\
(\mathrm{n}=7)\end{array}$ & \\
\hline Age, (years) & & & 1.000 \\
\hline$\leq 60$ & 24 & 6 & \\
\hline$>60$ & 6 & 1 & \\
\hline Gender & & & 0.570 \\
\hline Male & 26 & 7 & \\
\hline Female & 4 & 0 & \\
\hline HBsAg & & & 0.571 \\
\hline Negative & 6 & 0 & \\
\hline Positive & 24 & 7 & \\
\hline Cirrhosis & & & 0.327 \\
\hline Absent & 24 & 4 & \\
\hline Present & 6 & 3 & \\
\hline AFP, $\mu \mathrm{g} / 1$ & & & 0.360 \\
\hline$\leq 20$ & 7 & 3 & \\
\hline$>20$ & 23 & 4 & \\
\hline Tumor size (cm) & & & 0.002 \\
\hline$\leq 5$ & 6 & 6 & \\
\hline$>5$ & 24 & 1 & \\
\hline No. of tumor nodules & & & 0.213 \\
\hline Single & 21 & 3 & \\
\hline Multiple & 9 & 4 & \\
\hline Tumor encapsulation & & & 0.217 \\
\hline Well encapsulated & 17 & 6 & \\
\hline Poorly encapsulated & 13 & 1 & \\
\hline Microvascular invasion & & & 0.674 \\
\hline Negative & 16 & 5 & \\
\hline Positive & 14 & 2 & \\
\hline Portal lymphatic status & & & 1.000 \\
\hline No & 28 & 7 & \\
\hline Yes & 2 & 0 & \\
\hline
\end{tabular}

Fisher's exact test was used in these comparisons.

of cells. Dex was unable to induce differentiation when used alone (data not shown). In addition, AFP was also increased early during induction, which was consistent with previously reported results (26). Next, AFP levels decreased gradually accompanied by the elevated ALB and TF, and the occurrence of hepatocyte-like morphology.

CXCR7 is inversely correlated with HNF4 $\alpha$ upon induced differentiation. Based on the model of HCC differentiation in vitro, we further observed CXCR7 levels following induced differentiation by $1 \%$ DMSO and $40 \mathrm{ng} / \mathrm{ml}$ OSM. After 

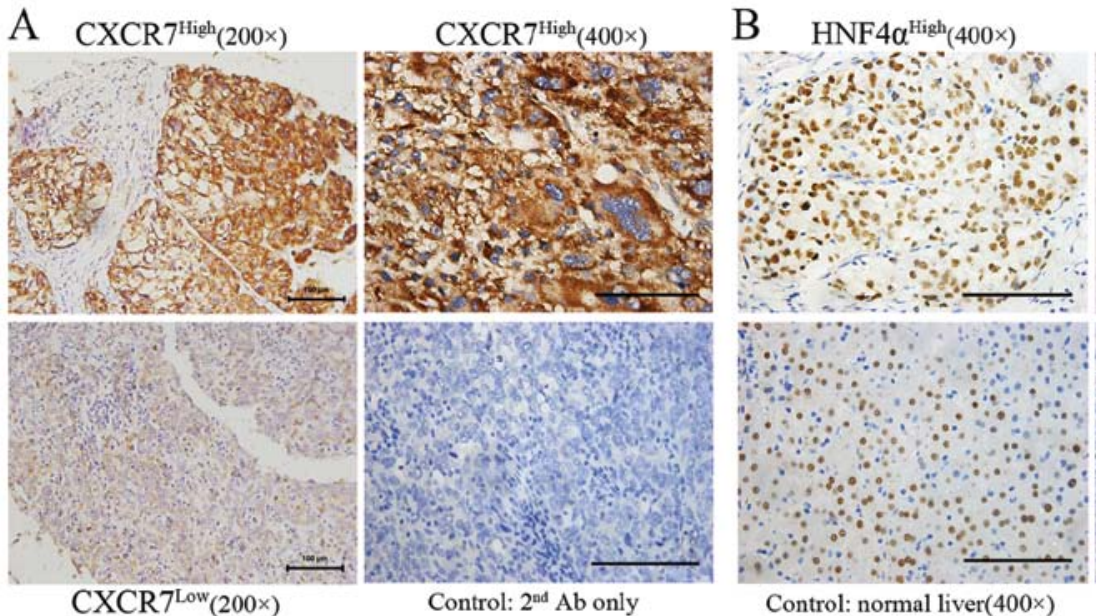

Control: normal liver(400x)

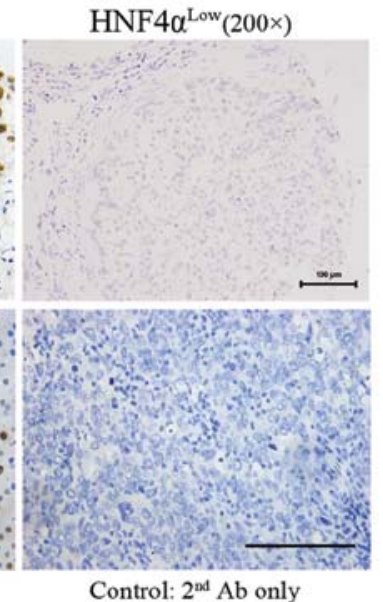

C
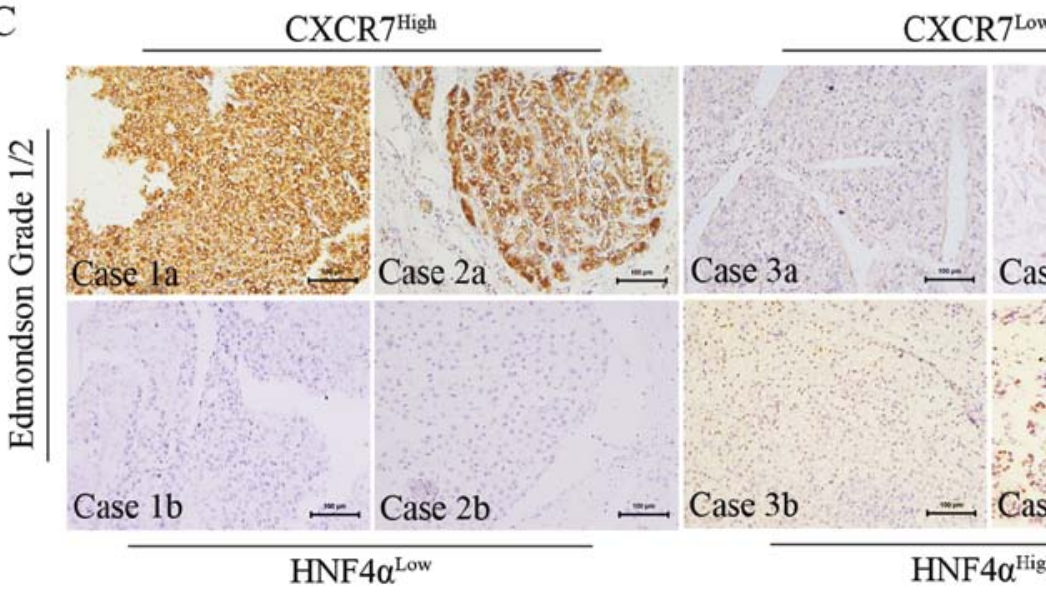

Control: $2^{\text {nd }} \mathrm{Ab}$ only
HNF4a $\alpha^{\text {Low }}$

HNF4 $\alpha^{\text {High }}$

Figure 4. Immunostaining of CXCR7 and HNF4 $\alpha$ in the TMA (A) Weak or strong staining was recognized as low or high CXCR7 expression, which mainly stained in the cytoplasm or on the surface of the tumor cells. (B) Low and high HNF4 $\alpha$ levels are shown. The staining of HNF4 $\alpha$ was mainly nuclear. The positive staining in normal hepatocytes indicated the specificity of the HNF4 $\alpha$ antibody. (C) IHC analysis in representative cases indicated that tumor tissues with high CXCR7 expression always had low HNF4 $\alpha$ expression, whereas CXCR7 ${ }^{\text {Low }}$ tissues were also likely to be HNF4 $\alpha^{\text {High }}$, particularly in the well-differentiated HCC cases. (x200 original magnification; scale bar, $100 \mu \mathrm{m}$ ).

DMSO treatment, CXCR7 expression was increased early and was decreased during the late stages of differentiation, which showed an inverse expression trend to $\mathrm{HNF} 4 \alpha$, which has a critical role in liver-specific gene expression. As shown in Fig. 3A, during early stages of induced differentiation, CXCR7 was elevated and the HNF4 $\alpha$ level was decreased in both Huh7 and HCCLM3 cells. Whereas during the late stage, CXCR7 levels were decreased and HNF4 $\alpha$ levels were elevated. In addition, CXCR7 levels were elevated while the opposite trend was observed for HNF4 $\alpha$ during the early stage of differentiation in response to $40 \mathrm{ng} / \mathrm{ml}$ OSM treatment (Fig. 3B).

High expression of CXCR7 is correlated with decreased $H N F 4 \alpha$ expression. Furthermore, IHC analysis of the TMA was performed to confirm the relationship between CXCR7 and $\mathrm{HNF} 4 \alpha$. Immunopositivity for CXCR7 was mainly observed at the membrane or in the cytoplasm of the HCC cells, whereas for HNF4 $\alpha$ it was expressed mainly in the nucleus. The expression intensity and localization of CXCR7 and $\mathrm{HNF} 4 \alpha$ are shown in Fig. 4A and B, respectively. Immunostaining of normal hepatocytes indicated the specificity and selectivity of anti-HNF $4 \alpha$. Stratum analysis based on cell differentiation (Edmondson grade $1 / 2 ; n=57$ ) indicated that CXCR7 was negatively correlated with $\mathrm{HNF} 4 \alpha$ expression (Spearman's rho $=-0.296 ; \mathrm{P}=0.025)$. Among the tumors with low HNF4 $\alpha$ expression $(n=48), 62.5 \%$ were $\mathrm{CXCR} 7^{\mathrm{High}}$, whereas only $22.2 \%$ of the tumors with high HNF4 $\alpha$ expression $(n=9)$ were $\mathrm{CXCR} 7^{\mathrm{High}}$. Furthermore, immunostaining analyses indicated that the distribution and expression levels of CXCR7 were inversely associated with those of HNF4 $\alpha$ on a relatively well-differentiated background (Fig. 4C). In addition, $\mathrm{CXCR} 7^{\text {High }} \mathrm{HNF} 4 \alpha^{\text {Low }}$ tumors tended to be larger (Fisher's exact test, $\mathrm{P}=0.002$ ), suggesting a relationship between differentiation and proliferation of HCC (Table I).

High expression of CXCR7 predicts poor prognosis when combined with $H N F 4 \alpha$. There was a statistically significant difference in $\mathrm{OS}$ between the $\mathrm{CXCR} 7^{\text {High }}$ and $\mathrm{CXCR} 7^{\text {Low }}$ groups on the $\mathrm{HNF} 4 \alpha^{\text {Low }}$ background (log-rank test; $\mathrm{P}=0.032$ ). In particular, there was a strong difference during the early stages of follow-up (Breslow test; $\mathrm{P}=0.007$; Fig. 5A). The median OS of $\mathrm{CXCR} 7^{\text {High }}$ patients was much shorter than that of CXCR7 $7^{\text {Low }}$ patients (36 vs. 81.2 months). Similarly, on the HNF $4 \alpha^{\text {Low }}$ background, the TTP of the CXCR $7^{\text {High }}$ patients was significantly shorter than that for the $\mathrm{CXCR} 7^{\text {Low }}$ patients (log-rank test; $\mathrm{P}=0.038$ ), particularly during the early stages 
A

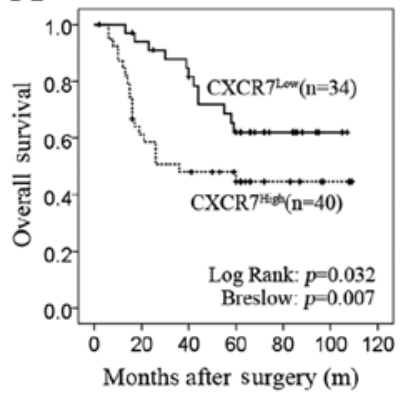

B

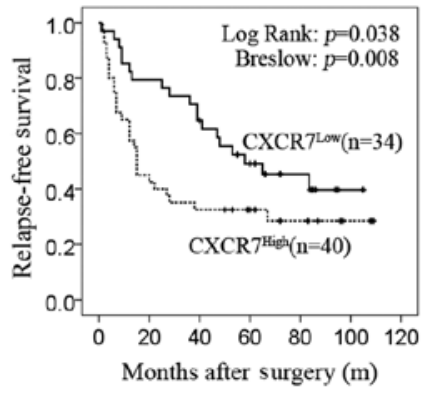

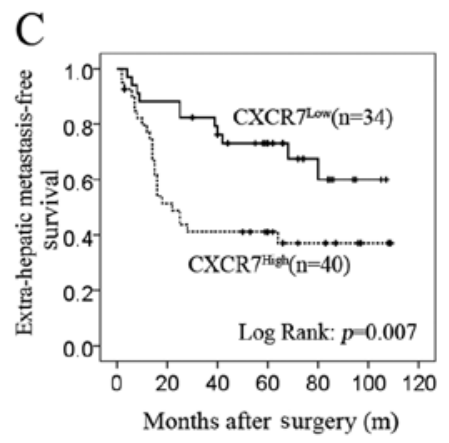

HNF $4 \alpha^{\text {Low }}$

D

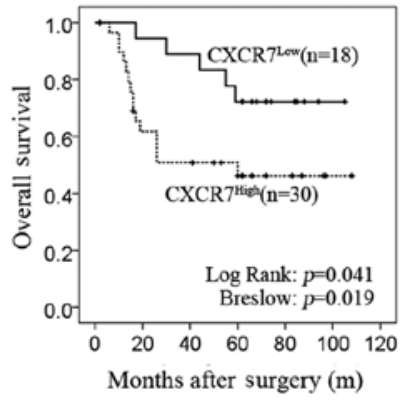

E

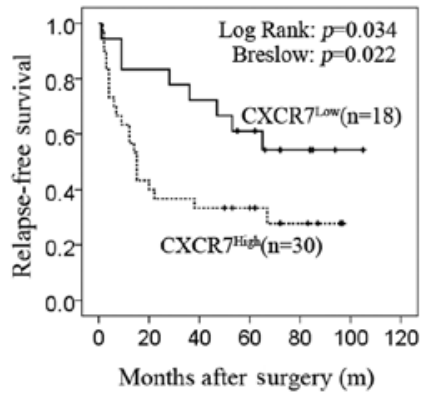

F

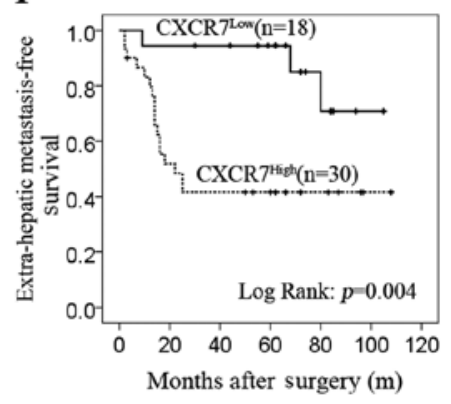

Edmondson Grade $1 / 2 \&$ HNF $4 \alpha^{\text {Low }}$

Figure 5. CXCR7 is a strong prognostic biomarker for poor survival when combined with low HNF4 $\alpha$ expression. In HNF4 $\alpha^{\text {Low }}$ HCC, high CXCR7 expression was correlated with (A) poor overall survival and (B) reduced time to progression, particularly during the early stages of follow-up. (C) In addition, extrahepatic metastases occurred more rapidly in patients with CXCR7 ${ }^{\mathrm{High}} \mathrm{HCC}$. Further stratification based on relative degree of differentiation indicated the prognostic value of CXCR7 in the survival of patients post-resection, including (D) overall survival, (E) time to progression and (F) time to metastasis.

A

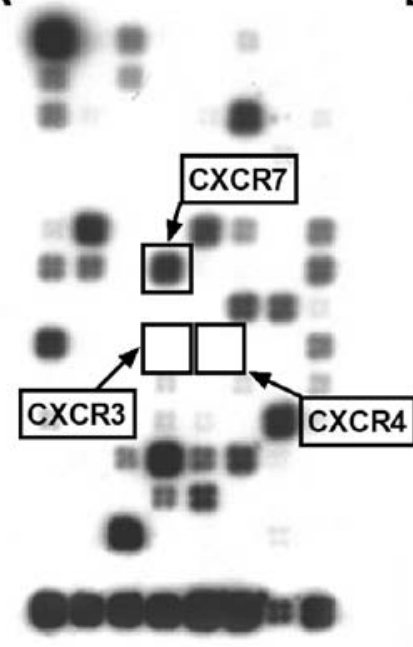

HCCLM3
B

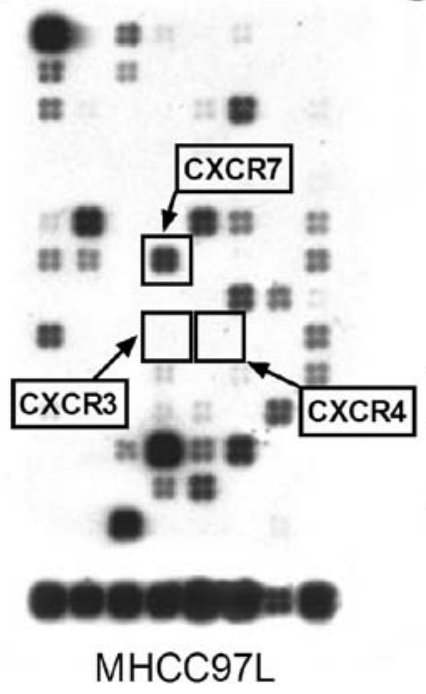

C

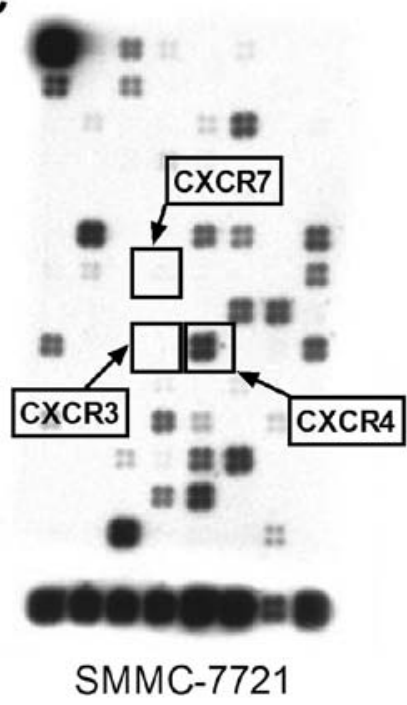

Figure 6. HCCLM3 is a CXCR7 ${ }^{+} \mathrm{CXCR}^{-} \mathrm{CXCR} 4^{-} \mathrm{HCC}$ cell line. Human chemokine and chemokine receptor GEArray kits were hybridized with cDNA probes derived from HCCLM3, MHCC97-L and SMMC-7721 cells. Strong CXCR7 and non-detectable CXCR3 and CXCR4 signals were observed for the HCCLM3 cell line.

of follow-up (Breslow test; $\mathrm{P}=0.008$; Fig. 5B). In addition, the time that relapsed before observing lung metastasis was shorter for CXCR7 ${ }^{\mathrm{High}}$ patients than for CXCR7 ${ }^{\text {Low }}$ patients (log-rank test; $\mathrm{P}=0.007$ ) (Fig. $5 \mathrm{C}$ ). In addition, in patients with high HNF4 $\alpha$ expression in tumors, there was no signifi- cant difference between $\mathrm{CXCR} 7^{\mathrm{High}}$ patients and $\mathrm{CXCR} 7^{\text {Low }}$ patients (data not shown).

Since there was a greater correlation between CXCR7 and $\mathrm{HNF} 4 \alpha$ in relatively well-differentiated $\mathrm{HCC}$, the prognostic value of CXCR7 was further evaluated in patients with 

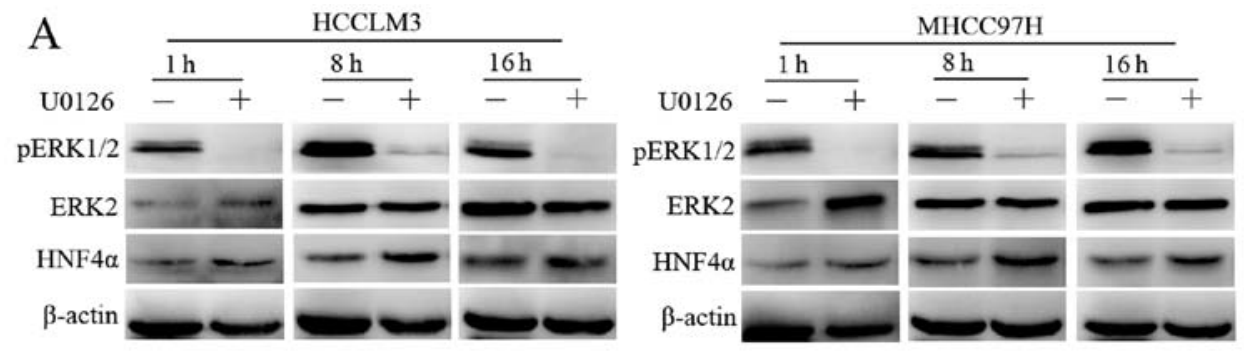

$\mathrm{B}$
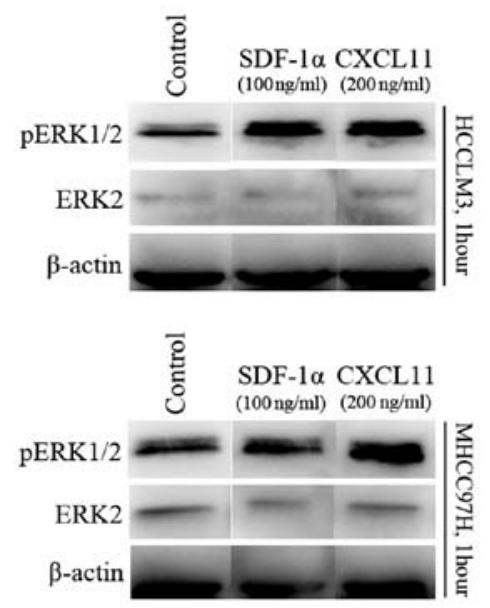

$\mathrm{C}$
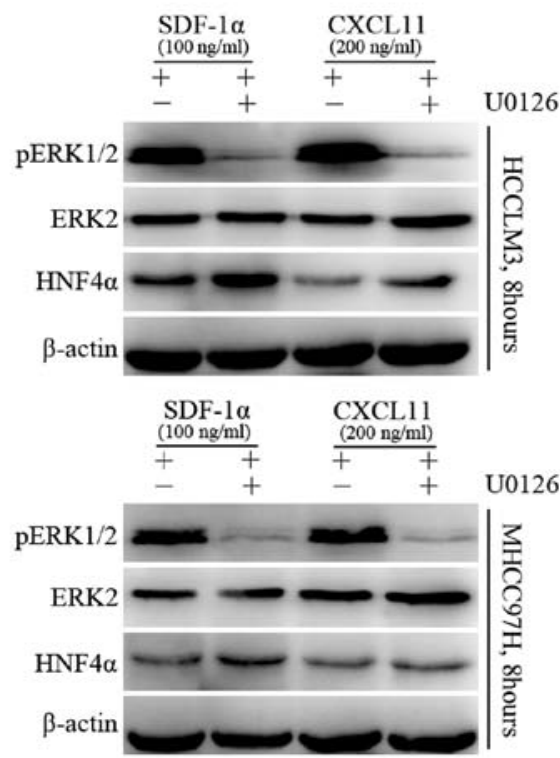

$\mathrm{E}$

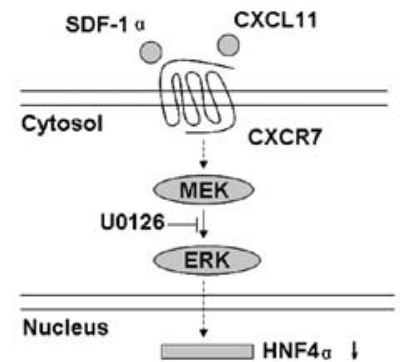

Figure 7. CXCR7 signaling suppresses HNF4 $\alpha$ expression in an ERK-dependent fashion in HCC. (A) HCCLM3 and MHCC97H cells were cultured with or without the MEK1/2 inhibitor U0126. Phosphorylation of MAPKs ERK1/2 was decreased at 1, 8 or $16 \mathrm{~h}$, and was accompanied by increased HNF4a expression, which indicates the suppression of the ERK pathway by HNF4 $\alpha$. (B) Cells were cultured in the presence of CXCR7 ligands (100 ng/ml SDF-1 $\alpha$ or $200 \mathrm{ng} / \mathrm{ml} \mathrm{CXCL11).} \mathrm{Elevated} \mathrm{phosphorylation} \mathrm{of} \mathrm{ERK1/2} \mathrm{was} \mathrm{observed} \mathrm{a} \mathrm{short} \mathrm{time} \mathrm{after} \mathrm{ligand} \mathrm{activation,} \mathrm{which} \mathrm{indicated} \mathrm{that} \mathrm{CXCR7} \mathrm{ligands} \mathrm{can} \mathrm{activate}$ ERK signaling. (C) HCCLM3 and MHCC97H cells were cultured in the presence of CXCR7 ligands (100 ng/ml SDF-1 $\alpha$ or $200 \mathrm{ng} / \mathrm{ml}$ CXCL11) with or without the MEK1/2 inhibitor U0126. Western blotting indicated the elevated HNF4 $\alpha$ level after using U0126, indicating that the use of U0126 abrogated the inhibitory effect of CXCR7 ligands on HNF4 $\alpha$ expression. (D) Knockdown of CXCR7 increased HNF4 $\alpha$ expression in HCC cells. C/EBP $\alpha$, another critical transcriptional factor, did not exhibit substantial changes. (E) Schematic drawing illustrating the proposed mechanism by which the transmembrane receptor CXCR7 mediates the ERK-dependent pathway to suppress HNF4 $\alpha$

Edmondson grade 1/2 HCC (n=48) (Fig. 5D and E). We found that CXCR7 expression had a strong prognostic value in the time-to-metastasis analysis (log-rank test; $\mathrm{P}=0.004$; Fig. 5F).

Activated CXCR7 suppresses HNF4 $\alpha$ through ERK-dependent signaling. Since CXCR7 is an atypical G-protein-coupled receptor that has been reported to mediate extracellular regulated protein kinase (ERK) signaling, we explored the possibility of a CXCR7-ERK-HNF4 $\alpha$ pathway. Gene chip analysis indicated that HCCLM3 cells highly expressed CXCR7. CXCR4 and CXCR3, however, were nearly undetectable (Fig. 6). Therefore, we utilized HCCLM3 cells as the
CXCR7 ${ }^{+}$CXCR4 ${ }^{-C X C R} 3^{-}$model to observe CXCR7 signaling stimulated by SDF-1 $\alpha$ and CXCL11. Phosphorylation of MAPKs ERK1/2 was decreased at 1,8 or $16 \mathrm{~h}$, and was accompanied by increased HNF4 $\alpha$ expression (Fig. 7A), which indicated suppression of the ERK pathway by HNF4 $\alpha$. Then, following stimulation with SDF-1 $\alpha$ or CXCL11, elevated phosphorylation of ERK1/2 was observed (Fig. 7B). Meanwhile, western blotting indicated elevated HNF4 $\alpha$ levels after exposure to U0126 (Fig. 7C), which indicated that the use of U0126 abrogated the inhibitory effect of CXCR7 ligands on HNF4 $\alpha$ expression. Furthermore, after downregulating CXCR7 in the HCCLM 3 cells, HNF4 $\alpha$ was increased in all positive siRNA 
groups (Fig. 7D). However, another hepatic-enriched nuclear factor $\mathrm{C} / \mathrm{EBP} \alpha$ was not obviously affected. Another cell line, MHCC97H, with the same genetic background as HCCLM3 and also expressing high levels of CXCR7 and extremely low levels of CXCR4 and CXCR3 was used to confirm these results. The results with this cell line were similar to those using the HCCLM3 cell line.

\section{Discussion}

As a chemokine receptor, CXCR7 plays roles in crest cell movement in development. Similarly, the role of CXCR7 in invasion and metastasis has been demonstrated in tumors. However, as an atypical G-protein-coupled receptor, other functions of CXCR7 have just begun to be unveiled. Evidence suggests the potential role of CXCR7 during the differentiation of embryonic stem cells (17). In the present study, the close correlation between CXCR7 and differentiation of HCC was found, including its opposite expression pattern with $\mathrm{HNF} 4 \alpha$ upon induced differentiation. Moreover, the relationship between CXCR7 and HNF4 $\alpha$ was confirmed by histological analysis of HCC samples after hepatic resection. Furthermore, high CXCR7 expression levels were closely correlated with poor survival and extrahepatic metastasis in tumors with low HNF4 $\alpha$ expression. These findings strongly suggest a critical role for CXCR7 in the differentiation of HCC.

Differentiation therapy is a useful therapeutic strategy for malignancy. However, effective differentiation therapy is lacking for HCC. In this study, a new pathway mediated by CXCR7 in de-differentiated HCC was demonstrated, as summarized in Fig. 7E. Although the screened HCC cell lines have distinct genetic backgrounds and different CSC-like biomarkers, the typical differentiation phenotype can be observed in each, indicating that the CXCR7-MAPK-HNF4 $\alpha$ cascade is the general pathway in the differentiation of HCC. In addition, adenoviral targeting of HNF4 $\alpha$ has achieved success in mouse models of HCC (4). Therefore, the CXCR7MAPK-HNF4 $\alpha$ pathway discovered in this study represents a promising target for differentiation therapy of HCC.

In this study, the suppression of HNF4 $\alpha$ by CXCR7 signaling was ERK-dependent, which was inhibited by a specific MEK inhibitor. Similar to our finding, the MAPK pathway has been found to control HNF4 $\alpha$ directly in hepatoma cells (27). Moreover, mucroporin-M1 was found to selectively activate MAPK signaling and lead to the downregulation of $\mathrm{HNF} 4 \alpha$ expression (28). On the other hand, as an atypical G-protein-coupled receptor, CXCR7 has been shown to mediate $\beta$-arrestin-biased signaling. As a polyfunctional adaptor molecule, $\beta$-arrestin can mediate multiple downstream pathways (29). Functionally, CXCR7 has been shown to activate MAPK through $\beta$-arrestin. Meanwhile, it is well known that the MAPK pathway is one of the main pathways to control differentiation (30). Therefore, $\beta$-arrestin may participate in the CXCR7-ERK-HNF4 $\alpha$ signaling cascade, which warrants further study.

In addition to our finding that $\mathrm{CXCR} 7$ controlled HNF4 $\alpha$ expression, HNF4 $\alpha$ can also be modified at the post-translational level (31). Additionally, cyclin D1 inhibits the function of HNF4 $\alpha$ (32), whereas GSK3 $\beta$ promotes the function of HNF4 $\alpha$ (33). In addition, TGF $\beta$ has been found to impair the efficiency of HNF4 $\alpha$ through GSK3 $\beta$ inactivation (34). Recently, the Wnt/ $\beta$-catenin pathway was reported to converge with $\mathrm{HNF} 4 \alpha$-driven transcription in liver zone specification (35). However, unlike these previous reports, our findings clearly suggest a signaling pathway involved in the differentiation of HCC.

The best proof of principle for differentiation therapy has been the treatment of acute promyelocytic leukemia with alltrans-retinoic acid. However, retinoic acid has a very limited role in $\mathrm{HCC}$ differentiation, which suggests that $\mathrm{HCC}$ has its own differentiation features. The HCC cell lines used in the present study have distinct genetic backgrounds and different CSC-like biomarkers. However, the selected HCC cell lines can be induced successfully to differentiate into hepatocyte-like cells, which suggests the existence of CSC-like cells or progenitor-like cells in HCC tumor cells and the possibility for differentiation therapy. In addition, upregulation of AFP and downregulation of $\mathrm{HNF} 4 \alpha$ were observed during the early stages of induced differentiation, similar to a report concerning the differentiation of hepatocytes from embryonic stem cells (26). These findings suggest that HCC cells reside in different de-differentiated stages. Additionally, BMP4 administration has been found to induce the differentiation of $\mathrm{CD}_{133^{+}}$hepatic CSCs and block their contributions to HCC. Therefore, differentiation therapy based on hepatic CSCs may be a promising strategy.

In conclusion, our data indicate that the transmembrane receptor CXCR7 is closely associated with the differentiation of HCC. Activated CXCR7 signaling can suppress the key transcriptional factor HNF4 $\alpha$ through ERK activation, implicating the CXCR7-ERK-HNF4 $\alpha$ cascade as a potential target for HCC differentiation therapy.

\section{Acknowledgements}

This study was supported by the State Key Project on Infectious Diseases of China (no. 2012ZX10002-016) and the Youth Backbone Fund from Fudan University (B-233).

\section{References}

1. Ker CG, Chen HY, Chen KS, et al: Clinical significance of cell differentiation in hepatocellular carcinoma. Hepatogastroenterology 50: 475-479, 2003.

2. Nowak D, Stewart D and Koeffler HP: Differentiation therapy of leukemia: 3 decades of development. Blood 113: 3655-3665, 2009.

3. Zhang L, Sun H, Zhao F, et al: BMP4 administration induces differentiation of $\mathrm{CD}_{133^{+}}$hepatic cancer stem cells, blocking their contributions to hepatocellular carcinoma. Cancer Res 72: 4276-4285, 2012

4. Yin C, Lin Y, Zhang X, et al: Differentiation therapy of hepatocellular carcinoma in mice with recombinant adenovirus carrying hepatocyte nuclear factor-4alpha gene. Hepatology 48: 1528-1539, 2008

5. Ishiyama T, Kano J, Minami Y, Iijima T, Morishita $Y$ and Noguchi M: Expression of HNFs and C/EBP alpha is correlated with immunocytochemical differentiation of cell lines derived from human hepatocellular carcinomas, hepatoblastomas and immortalized hepatocytes. Cancer Sci 94: 757-763, 2003.

6. Costa RH, Kalinichenko VV, Holterman AX and Wang X: Transcription factors in liver development, differentiation, and regeneration. Hepatology 38: 1331-1347, 2003.

7. DeLaForest A, Nagaoka M, Si-Tayeb K, et al: HNF4A is essential for specification of hepatic progenitors from human pluripotent stem cells. Development 138: 4143-4153, 2011. 
8. Walesky C, Edwards G, Borude P, et al: Hepatocyte nuclear factor 4 alpha deletion promotes diethylnitrosamine-induced hepatocellular carcinoma in mice. Hepatology 57: 2480-2490, 2013.

9. Ning BF, Ding J, Yin C, et al: Hepatocyte nuclear factor 4 alpha suppresses the development of hepatocellular carcinoma. Cancer Res 70: 7640-7651, 2010.

10. Balabanian K, Lagane B, Infantino S, et al: The chemokine SDF-1/CXCL12 binds to and signals through the orphan receptor RDC1 in T lymphocytes. J Biol Chem 280: 3576035766, 2005

11. Burns JM, Summers BC, Wang Y, et al: A novel chemokine receptor for SDF-1 and I-TAC involved in cell survival, cell adhesion, and tumor development. J Exp Med 203: 2201-2213, 2006.

12. Rajagopal S, Kim J, Ahn S, et al: Beta-arrestin - but not $\mathrm{G}$ protein-mediated signaling by the 'decoy' receptor CXCR7. Proc Natl Acad Sci USA 107: 628-632, 2010.

13. Yu S, Crawford D, Tsuchihashi T, Behrens TW and Srivastava D The chemokine receptor CXCR7 functions to regulate cardiac valve remodeling. Dev Dyn 240: 384-393, 2011.

14. Maksym RB, Tarnowski M, Grymula K, et al: The role of stromal-derived factor-1-CXCR7 axis in development and cancer. Eur J Pharmacol 625: 31-40, 2009.

15. Xue TC, Chen RX, Han D, et al: Down-regulation of CXCR7 inhibits the growth and lung metastasis of human hepatocellular carcinoma cells with highly metastatic potential. Exp Ther Med 3: 117-123, 2012

16. Xue TC, Chen RX, Ren ZG, Zou JH, Tang ZY and Ye SL: Transmembrane receptor CXCR7 increases the risk of extrahepatic metastasis of relatively well-differentiated hepatocellular carcinoma through upregulation of osteopontin. Oncol Rep 30 105-110, 2013.

17. Pasini D, Bracken AP, Hansen JB, Capillo M and Helin K: The polycomb group protein Suz12 is required for embryonic stem cell differentiation. Mol Cell Biol 27: 3769-3779, 2007.

18. Dalby B, Cates S, Harris A, et al: Advanced transfection with Lipofectamine 2000 reagent: primary neurons, siRNA, and high-throughput applications. Methods 33: 95-103, 2004.

19. Sun HC, Zhuang PY, Qin LX, et al: Incidence and prognostic values of lymph node metastasis in operable hepatocellular carcinoma and evaluation of routine complete lymphadenectomy. J Surg Oncol 96: 37-45, 2007.

20. Simon R, Mirlacher M and Sauter G: Immunohistochemical analysis of tissue microarrays. Methods Mol Biol 664: 113-126, 2010.

21. Lugli A, Spichtin H, Maurer R, et al: EphB2 expression across 138 human tumor types in a tissue microarray: high levels of expression in gastrointestinal cancers. Clin Cancer Res 11: 6450-6458, 2005.

22. Liu LL, Fu D, Ma Y and Shen XZ: The power and the promise of liver cancer stem cell markers. Stem Cells Dev 20: 2023-2030, 2011.
23. Lee TK, Castilho A, Cheung VC, Tang KH, Ma S and Ng IO: $\mathrm{CD} 24(+)$ liver tumor-initiating cells drive self-renewal and tumor initiation through STAT3-mediated NANOG regulation. Cell Stem Cell 9: 50-63, 2011.

24. Yang ZF, Ho DW, Ng MN, et al: Significance of CD90 $0^{+}$cancer stem cells in human liver cancer. Cancer Cell 13: 153-166, 2008.

25. Yamashita T, Honda M, Nio $\mathrm{K}$, et al: Oncostatin $\mathrm{m}$ renders epithelial cell adhesion molecule-positive liver cancer stem cells sensitive to 5- Fluorouracil by inducing hepatocytic differentiation. Cancer Res 70: 4687-4697, 2010.

26. Hay DC, Zhao D, Fletcher J, et al: Efficient differentiation of hepatocytes from human embryonic stem cells exhibiting markers recapitulating liver development in vivo. Stem Cells 26: 894-902, 2008.

27. Hatzis P, Kyrmizi I and Talianidis I: Mitogen-activated protein kinase-mediated disruption of enhancer-promoter communication inhibits hepatocyte nuclear factor 4alpha expression. Mol Cell Biol 26: 7017-7029, 2006.

28. Zhao Z, Hong W, Zeng Z, et al: Mucroporin-M1 inhibits hepatitis $B$ virus replication by activating the mitogen-activated protein kinase (MAPK) pathway and down-regulating HNF4alpha in vitro and in vivo. J Biol Chem 287: 30181-30190, 2012.

29. Shenoy SK and Lefkowitz RJ: $\beta$-Arrestin-mediated receptor trafficking and signal transduction. Trends Pharmacol Sci 32: 521-533, 2011.

30. Basson MA: Signaling in cell differentiation and morphogenesis. Cold Spring Harb Perspect Biol 4: 2012 pii: a008151. doi: 10.1101/cshperspect.a008151.

31. Yokoyama A, Katsura S, Ito R, et al: Multiple post-translational modifications in hepatocyte nuclear factor $4 \alpha$. Biochem Biophys Res Commun 410: 749-753, 2011.

32. Hanse EA, Mashek DG, Becker JR, et al: Cyclin D1 inhibits hepatic lipogenesis via repression of carbohydrate response element binding protein and hepatocyte nuclear factor $4 \alpha$. Cell Cycle 11: 2681-2690, 2012.

33. Sakamaki J, Daitoku H, Kaneko Y, Hagiwara A, Ueno K and Fukamizu A: GSK3 $\beta$ regulates gluconeogenic gene expression through HNF4 $\alpha$ and FOXO1. J Recept Signal Transduct Res 32: 96-101, 2012

34. Cozzolino AM, Alonzi T, Santangelo L, et al: TGF $\beta$ overrides HNF $4 \alpha$ tumor suppressing activity through GSK $3 \beta$ inactivation: implication for hepatocellular carcinoma gene therapy. J Hepatol 58: 65-72, 2013.

35. Colletti M, Cicchini C, Conigliaro A, et al: Convergence of Wnt signaling on the HNF4alpha-driven transcription in controlling liver zonation. Gastroenterology 137: 660-672, 2009. 